ISSN: 2320-7167

Volume 10 Issue 02 February 2022, Page no. - 2564-2572

Index Copernicus ICV: 57.55, Impact Factor: 7.184

DOI: 10.47191/ijmcr/v10i2.03

\title{
Dynamical System Stability Analysis of the Interaction Nitrogen, Phytoplankton, Macrobenthos, and Sediment with Gradient Variable Method
}

\author{
Tri Yastuti Laksanahati ${ }^{1}$, Widowati ${ }^{2}$, Sapto Purnomo Putro ${ }^{3}$, Satriyo Adhy ${ }^{4}$, Mohammad Helmi ${ }^{5}$, Rita Rostika ${ }^{6}$ \\ ${ }^{1,2}$ Departement of Mathematics, Faculty of Science and Mathematics, Diponegoro University, Jl. Prof Soedarto SH, Semarang \\ 50275, Central Java, Indonesia \\ ${ }^{3}$ Departement of Biology, Faculty of Science and Mathematics, Diponegoro University, J1. Prof Soedarto SH, Semarang 50275, \\ Central Java, Indonesia \\ ${ }^{4}$ Departement of Informatics, Faculty of Science and Mathematics, Diponegoro University, Jl. Prof Soedarto SH, Semarang \\ 50275, Central Java, Indonesia \\ ${ }_{5}^{5}$ Department of Oceanography, Faculty of Fishery and Marine Science, Diponegoro University, Semarang, \\ Indonesia \\ ${ }^{6}$ Department of Fishery, Faculty of Fishery and Marine Science, Padjadjaran University, Bandung, Indonesia
}

\begin{tabular}{ll}
\hline ARTICLE INFO & ABSTRACT \\
\hline $\begin{array}{l}\text { Published online } \\
\text { 09 February 2022 }\end{array}$ & $\begin{array}{l}\text { In the shrimp pond aquaculture system, metabolic waste in the form of unfeed pellets and shrimp } \\
\text { excretion can reduce water quality and increase both soluble organic matter in waters and organic } \\
\text { deposition to sediments. A developed dynamical system was formed based on the interactions that } \\
\text { occur between phytoplankton growth, macrobenthos density, and sediment in response to nitrogen } \\
\text { and phosphate concentrations. The purpose of this research was to analize the stability of the } \\
\text { proposed dynamical system. Furthermore, we determine the level of water quality in the aquaculture } \\
\text { system based on the interaction between four variables, i.e. nitrogen, abundance of phytoplankton, } \\
\text { abundance of macrobenthos, and sediment properties. The Lyapunov stability theory using the } \\
\text { Gradient Variable method was used to determine the global stability of the systems. Assumption of } \\
\text { this method is determine } V \text { and } \nabla V \text { for gradient variable function. In the Lyapunov function, if the } \\
\text { value of the scalar function is definite positive and its first derivative is definite negative, then the } \\
\text { system is globally asymptotically stable. Based on mathematical analysis and numerical simulation, } \\
\text { the shrimp pond dynamical system model in BBPBAP Jepara, Indonesia was globally } \\
\text { asymptotically stable at equilibrium point. }\end{array}$ \\
Corresponding author: \\
Widowati
\end{tabular}

KEYWORDS: Shrimp pond, Dynamical system, Stability, Lyapunov, Gradient Variable

\section{INTRODUCTION}

The world's aquaculture cultivation faces many challenges due to over exploitation[1]. Based on data FAO, increasing demand for aquatic products depends on the growth of aquaculture. It is expected that the growth of aquaculture cultivation can increase from $44 \%$ from 167 million tons of production in 2013 to $52 \%$ from 196 million tons in 2025. In addition, FAO [2] predicts that by 2025, developing countries, especially Asian countries, will remain the main producers of aquaculture.
One of the cultivations in aquaculture is shrimp farming. In general, shrimp farming produces an abundance of organic waste and at the same time reduces the nutrients for shrimp. Only less than $1 / 5$ of the nitrogen is converted to shrimp biomass, while more than $1 / 3$ to organic waste [3], [4]. One of the organic wastes in aquaculture is nitrogen. Nitrogen is important in dynamic aquaculture systems because it is both a nutrient and a poison. The dynamic nitrogen system of shrimp pond culture has been studied using mathematical modeling [5]-[7]. Furthermore, uptake by phytoplankton and sedimentation are the main processes in the dynamic 
system of the nitrogen cycle in shrimp ponds with low water exchange rates [6]. Subsequent studies have investigated the remineralization of precipitated nitrogen [8], [9].

Many studies have been developed on nitrogen in aquatic systems. One of them is the modeling of dynamical nitrogen systems in shrimp intensive ponds [10]. This model describes the processes that affect the nitrogen cycle, namely ammonia, nitrate, nitrite, phytoplankton, and sediment. The model was developed by separating the concentrations of nitrate and nitrite [11]. A model was developed by adding an additional variable, namely phosphate concentration [12].

In addition to producing organic waste, aquaculture systems also have an impact on the benthic environment [13]. In the study [13], the presence of organic waste will affect the nutrient cycle in the benthic environment and the relationship between sediment quality and the presence of benthic macrofauna in shrimp farming. Benthic macrofauna or so-called macrobenthos are basic aquatic organisms, in the form of animals and plants that live on the bottom surface or at the bottom of the waters [14]. Research [15] showed that the organic matter content was directly proportional to the abundance of macrobenthos. The more organic waste in the waters, the more the abundance of macrobenthos increases. Subsequent research on the relationship between macrobenthos abundance and water quality [16].

This study focuses on the interaction of the dynamic nitrogen system model and the density of macrobenthos in shrimp culture in ponds. In this dynamic system model, a new variable is added, namely the density of macrobenthos. The purpose of this study was to determine the dynamical stability analysis system of nitrogen, phytoplankton, macrobenthos, and sediment in shrimp culture in ponds by constructing the lyapunov function using the gradient method.

\section{DEVELOPED MATHEMATICAL MODELING}

In the previous research on shrimp cultivation systems [12] a dynamic system model of the interaction between ammonia, nitrate, nitrite, phytoplankton, and sediment has been given. In the sediment there are macrobenthos which are living organisms, in the form of animals and plants. Macrobenthos has various important roles, including; as an important part in food webs, aquatic ecosystems, improving sediment structure, and as a bioindicator of water quality [14]. The interaction between nitrogen, phytoplankton, macrobenthos, and sediment will be made in a dynamic system model. Based on the dynamic system model of the waters, global stability will be analyzed by building the Lyapunov function using the Gradient Variable method. The Lyapunov function is applied to analyze the stability of both linear and nonlinear systems, time-invariant systems and time-varrying systems [17]. The process of mathematical modeling between nitrogen, phytoplankton, macrobenthos, and sediment is given in Figure 1.

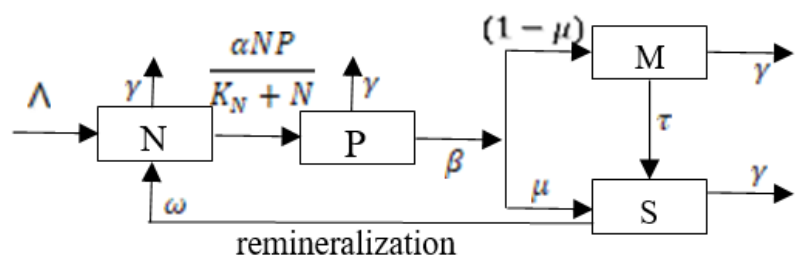

Figure 1. The process of mathematical modeling between nitrogen, phytoplankton, macrobenthos, and sediment

The unfeed pellets and shrimp excretion are the main nutrients in shrimp cultivation. The organic matter on the waters as nitrogen input $(\Lambda)$, which is increasing the rate of concentration nitrogen. In addition, the rate of concentration nitrogen increased due to sediment remineralization $(\omega S)$. The rate of concentration nitrogen will be reduced by $\frac{\alpha N P}{K_{N}+N}$ as a nutrient for phytoplankton. The concentration nitrogen in the waters will release for $\gamma N$. The rate of concentration nitrogen at time $\mathrm{t}$ is obtained:

$$
\frac{d N}{d t}=\Lambda+\omega S-\gamma N-\frac{\alpha N P}{K_{N}+N}
$$

The growth rate of phytoplankton is influenced by the formation of concentration nitrogen. The form of Michelis Menten is used as nitrogen formation, namely $\frac{N}{K_{N}+N}$. Biomass of phytoplankton increased due to the uptake of nitrogen as a phytoplankton nutrient. Furthermore, the biomass of phytoplankton decreased due to the release of phytoplankton in the waters $(\gamma P)$, and the death of phytoplankton $(\beta P)$. Thus, the following equation:

$$
\frac{d P}{d t}=\frac{\alpha N P}{K_{N}+N}-(\beta+\gamma) P
$$

Biomass of macrobenthos increased due to the food consumed $(1-\mu) P$. Meanwhile, the biomass of macrobenthos decreased due to sedimentation $(\tau M)$ and the release rate in the waters $(\gamma M)$. Thus, the macrobenthos growth rate was obtained as follows:

$$
\frac{d M}{d t}=\beta(1-\mu) P-(\gamma+\tau) M
$$

The increased of sediment rate is due to the death of phytoplankton and the death of macrobenthos. The mortality of phytoplankton and macrobenthos, respectively, was $\beta \mu P$ and $\tau M$. Meanwhile, the decreased in sediment due to remineralization $(\omega S)$. It is obtained that the rate of change of sediment: 


$$
\frac{d S}{d t}=\beta \mu P+\tau M-\omega S
$$

Based on equations (1) - (4), the interaction model of the phytoplankton, nitrogen, macrobenthos, and sediment on the shrimp cultivation obtained:

$$
\begin{aligned}
& \frac{d P}{d t}=\frac{\alpha N P}{K_{N}+N}-(\beta+\gamma) P \\
& \frac{d N}{d t}=\Lambda+\omega S-\gamma N-\frac{\alpha N P}{K_{N}+N} \\
& \frac{d M}{d t}=\beta(1-\mu) P-(\gamma+\tau) M \\
& \frac{d S}{d t}=\beta \mu P+\tau M-\omega S
\end{aligned}
$$

where $P, N, M, S$ are phytoplankton, nitrogen, macrobenthos, and sediment, respectively at $t$ is time.

\section{STABILITY ANALYSIS BY USING GRADIENT VARIABLE METHOD}

The Lyapunov function can be used to analyze the stability of dynamical system. One of method that can construct the Lyapunov function is the gradient variable method. In the following, the theorem regarding the stability of dynamical systems is given.

Theorem 1. [18]

Suppose that $x=0$ be an equilibrium point for $\dot{x}=f(x)$ and $D \subset R^{n}$ be a domain containing $x=0$. Suppose that $V: D \rightarrow R$ be a continuously differentiable function such that

$$
\begin{aligned}
& V(0)=0 \text { and } V(x)>0, \forall_{x} \neq 0 \\
& \dot{V}(x) \leq 0, \forall_{x} \neq 0
\end{aligned}
$$

Then $x=0$ is stable. Moreover, if $\dot{V}(x)<0, \forall_{x} \neq 0$ then $x=0$ is asymptotically stable.

\section{Proof:}

Let $\varepsilon>0$, with $r \in(0, \varepsilon]$ such that $B_{r}=\left\{x \in R^{n}\|x\| \leq r\right\} \subset D$. Suppose $\alpha=\min _{\|x\|=r} V(x)$. Then, $\alpha>0$ by $V(0)=0$ and $V(x)>0, \forall_{x} \neq 0$. Take $\quad \beta \in(0, \alpha) \quad$ and let $\Omega_{\beta}=\left\{x \in B_{r} \mid V(x) \leq \beta\right\}$. Then, $\Omega_{\beta}$ is in the interior of $B_{r}$. The set $\Omega_{\beta}$ has the property that any trajectory starting in $\Omega_{\beta}$ at point $t=0$ stays in $\Omega_{\beta}$ for all $t \geq 0$. This follows

$\dot{V}(x(t)) \leq 0 \Rightarrow V(x(t)) \leq V(x(0)) \leq \beta, \forall_{t} \geq 0$

$V(x)$ is continuous and $V(0)=0$, there is $\delta>0$ such that $\|x\| \leq \delta \Rightarrow V(x)<\beta \quad$ then, $\quad B_{\delta} \subset \Omega_{\beta} \subset B_{r} \quad$ and $x(0) \in B_{\delta} \Rightarrow x(0) \in \Omega_{\beta} \Rightarrow x(t) \in \Omega_{\beta} \Rightarrow x(t) \in B_{r}$. Therefore, $\|x(0)\|<\delta \Rightarrow\|x(t)\|<r \leq r \leq \varepsilon, \forall_{t} \geq 0 \quad$ which shows that the equilibrium point $x=0$ is stable. To denote asymptotic stability, showed that $x(t) \rightarrow 0$ as $t \rightarrow \infty, \forall_{\alpha}>0$, there is
$T>0$ such that $\|x(t)\|<\alpha, \forall_{t}>T$. Since $V(x(t))$ is monotonically decreasing and bounded from below by zero, $V(x(t)) \rightarrow c \geq 0$ as $t \rightarrow \infty$. Used a contradiction argument to show that $c=0$. Let $c>0$ and by continuity of $V(x)$, there is $d>0$ such that $B_{d} \subset \Omega_{c}$. The limit $V(x(t)) \rightarrow c>0$ implies that the trajectory $x(t)$ lies outside the ball $B_{d}$ for $t \geq 0$. Let $-\gamma=\max _{d \leq\|x\| \leq r} \dot{V}(x)$, which exists because the continuous function $\dot{V}(x)$ has a maximum over the compact set $\{d \leq\|x\| \leq r\}$. Based on $\dot{V}(x)<0, \forall_{x} \neq 0$, so $-\gamma<0$. It follows that

$$
V(x(t))=V(x(0))+\int_{0}^{t} \dot{V}(x(\tau)) d \tau \leq V(x(0))-\gamma t
$$

Since the right hand side will become negative, the inequality contradicts the assumption that $c>0$.

Based on the system equation (5), we can construct the linear equation:

$$
\begin{aligned}
& \dot{x}=f(x, t)=\left[\begin{array}{c}
\dot{P} \\
\dot{N} \\
\dot{M} \\
\dot{S}
\end{array}\right], \text { we can obtained } \\
& f=\left[\begin{array}{c}
f_{1} \\
f_{2} \\
f_{3} \\
f_{4}
\end{array}\right]=\left[\begin{array}{c}
\dot{P} \\
\dot{N} \\
\dot{M} \\
\dot{S}
\end{array}\right]=\left[\begin{array}{c}
\frac{\alpha N P}{K_{N}+N}-(\beta+\gamma) P \\
\Lambda+\omega S-\gamma N-\frac{\alpha N P}{K_{N}+N} \\
\beta(1-\mu) P-(\gamma+\tau) M \\
\beta \mu P+\tau M-\omega S
\end{array}\right]
\end{aligned}
$$

Based on the system equation (5), the Lyapunov function will be constructed using gradient method [18].

$\nabla V=\left[\begin{array}{llll}a_{11} & a_{12} & a_{13} & a_{14} \\ a_{21} & a_{22} & a_{23} & a_{24} \\ a_{31} & a_{32} & a_{33} & a_{34} \\ a_{41} & a_{42} & a_{43} & a_{44}\end{array}\right]\left[\begin{array}{c}P \\ N \\ M \\ S\end{array}\right]$,

$\operatorname{matrix~} \mathrm{A}=\left[\begin{array}{llll}a_{11} & a_{12} & a_{13} & a_{14} \\ a_{21} & a_{22} & a_{23} & a_{24} \\ a_{31} & a_{32} & a_{33} & a_{34} \\ a_{41} & a_{42} & a_{43} & a_{44}\end{array}\right]$

is a matrix symmetrical positive definite.

Based on the system equation (5), the differential function $V$ can be constructed from $\nabla V$

$$
\begin{aligned}
& \dot{V}(x)=\nabla V^{T} f \\
& \dot{V}(x)=\left[\begin{array}{c}
a_{11} P+a_{12} N+a_{13} M+a_{14} S \\
a_{21} P+a_{22} N+a_{23} M+a_{24} S \\
a_{31} P+a_{32} N+a_{33} M+a_{34} S \\
a_{41} P+a_{42} N+a_{43} M+a_{44} S
\end{array}\right]^{T}\left[\begin{array}{c}
\frac{\alpha N P}{K_{N}+N}-(\beta+\gamma) P \\
\Lambda+\omega S-\gamma N-\frac{\alpha N P}{K_{N}+N} \\
\beta(1-\mu) P-(\gamma+\tau) M \\
\beta \mu P+\tau M-\omega S
\end{array}\right]
\end{aligned}
$$


"Dynamical System Stability Analysis of the Interaction Nitrogen, Phytoplankton, Macrobenthos, and Sediment with Gradient Variable Method"

$$
\begin{aligned}
& =\left(a_{11} P+a_{12} N+a_{13} M+a_{14} S\right)\left(\frac{\alpha N P}{K_{N}+N}-(\beta+\gamma) P\right) \\
& +\left(a_{21} P+a_{22} N+a_{23} M+a_{24} S\right)\left(\Lambda+\omega S-\gamma N-\frac{\alpha N P}{K_{N}+N}\right) \\
& +\left(a_{31} P+a_{32} N+a_{33} M+a_{34} S\right)(\beta(1-\mu) P-(\gamma+\tau) M) \\
& +\left(a_{41} P+a_{42} N+a_{43} M+a_{44} S\right)(\beta \mu P+\tau M-\omega S)
\end{aligned}
$$

That obtained $V(x)$ is negative definite function

If $a_{11}, a_{12}, a_{13}, a_{14}>0, a_{21}, a_{22}, a_{23}, a_{24}>0$

$a_{31}, a_{32}, a_{33}, a_{34}>0, a_{41}, a_{42}, a_{43}, a_{44}>0$.

The qurl equations are

$$
\begin{aligned}
\frac{\partial \nabla V_{1}}{\partial N}=\frac{\partial \nabla V_{2}}{\partial P} \Rightarrow a_{12}=a_{21} & \frac{\partial \nabla V_{1}}{\partial M}=\frac{\partial \nabla V_{4}}{\partial P} \Rightarrow a_{14}=a_{41} \\
\frac{\partial \nabla V_{1}}{\partial F}=\frac{\partial \nabla V_{3}}{\partial P} \Rightarrow a_{13}=a_{31} & \frac{\partial \nabla V_{3}}{\partial M}=\frac{\partial \nabla V_{4}}{\partial F} \Rightarrow a_{34}=a_{43} \\
\frac{\partial \nabla V_{2}}{\partial F}=\frac{\partial \nabla V_{3}}{\partial N} \Rightarrow a_{23}=a_{32} & \frac{\partial \nabla V_{2}}{\partial M}=\frac{\partial \nabla V_{4}}{\partial N} \Rightarrow a_{24}=a_{42}
\end{aligned}
$$

The qurl equations are satisfied, so the $\bar{V}$ can be computed:

$$
\begin{aligned}
& V(x)=\int \nabla V \cdot d x \\
& =\int_{0}^{P} \nabla V_{1} \cdot d P+\int_{0}^{N} \nabla V_{2} \cdot d N+\int_{0}^{M} \nabla V_{3} \cdot d M+\int_{0}^{S} \nabla V_{4} \cdot d S \\
& =\int_{0}^{P} a_{11} P+a_{12} N+a_{13} M+a_{14} S \cdot d P+ \\
& \int_{0}^{N} a_{21} P+a_{22} N+a_{23} M+a_{24} S . d N \\
& +\int_{0}^{M} a_{31} P+a_{32} N+a_{33} M+a_{34} S . d M \\
& +\int_{0}^{S} a_{41} P+a_{42} N+a_{43} M+a_{44} S . d S \\
& =\frac{1}{2} a_{11} P^{2}+a_{12} N P+a_{13} M P+a_{14} S P \\
& +\frac{1}{2} a_{22} N^{2}+a_{21} P N+a_{23} M N+a_{24} S N \\
& +\frac{1}{2} a_{33} M^{2}+a_{31} P M+a_{32} N M+a_{34} S M \\
& +\frac{1}{2} a_{44} S^{2}+a_{41} P S+a_{42} N S+a_{43} M S \\
& V(x)=\frac{1}{2} a_{11} P^{2}+\frac{1}{2} a_{22} N^{2}+\frac{1}{2} a_{33} M^{2}+\frac{1}{2} a_{44} S^{2} \\
& +2 a_{12} P N+2 a_{13} P M+2 a_{14} P S \\
& +2 a_{23} M N+2 a_{24} S N+2 a_{34} S M
\end{aligned}
$$

Then, determined the positive definite matrix equation for the Lyapunov function as follows

$V(x)=x^{T} P x$

$$
\begin{aligned}
& =\left[\begin{array}{llll}
P & N & M & S
\end{array}\right]\left[\begin{array}{l}
A_{11} P+A_{12} N+A_{13} M+A_{14} S \\
A_{21} P+A_{22} N+A_{23} M+A_{24} S \\
A_{31} P+A_{32} N+A_{33} M+A_{34} S \\
A_{41} P+A_{42} N+A_{43} M+A_{44} S
\end{array}\right] \\
& V(x)=A_{11} P^{2}+A_{12} P N+A_{13} P M+A_{14} P S \\
& +A_{22} N^{2}+A_{21} N P+A_{23} N M+A_{24} N S \\
& +A_{33} M^{2}+A_{31} M P+A_{32} M N+A_{34} M S \\
& +A_{44} S^{2}+A_{41} S P+A_{42} S N+A_{43} S M \\
& V(x)=A_{11} P^{2}+A_{22} N^{2}+A_{33} M^{2}+A_{44} S^{2} \\
& +2 A_{12} P N+2 A_{13} P M+2 A_{14} P S \\
& +2 A_{23} N M+2 A_{24} N S+2 A_{34} M S
\end{aligned}
$$

Equations (6) and (7) are same, so we have

$$
\begin{aligned}
& V(x)= \frac{1}{2} a_{11} P^{2}+\frac{1}{2} a_{22} N^{2}+\frac{1}{2} a_{33} M^{2}+\frac{1}{2} a_{44} S^{2} \\
&+2 a_{12} P N+2 a_{13} P M+2 a_{14} P S \\
&+2 a_{23} M N+2 a_{24} S N+2 a_{34} S M \\
&=A_{11} P^{2}+A_{22} N^{2}+A_{33} M^{2}+A_{44} S^{2} \\
&+2 A_{12} P N+2 A_{13} P M+2 A_{14} P S \\
&+2 A_{23} N M+2 A_{24} N S+2 A_{34} M S
\end{aligned}
$$

Than we o btained

$$
\begin{aligned}
& A_{11}=\frac{1}{2} a_{11} ; A_{22}=\frac{1}{2} a_{22} ; A_{33}=\frac{1}{2} a_{33} ; A_{44}=\frac{1}{2} a_{44} ; \\
& A_{12}=a_{12} ; A_{13}=a_{13} ; A_{14}=a_{14} ; A_{23}=a_{23} ; A_{24}=a_{24} ; A_{34}=a_{34}
\end{aligned}
$$

So, we obtained matrix $\mathrm{A}$

$$
A=\left[\begin{array}{cccc}
\frac{1}{2} a_{11} & a_{12} & a_{13} & a_{14} \\
a_{12} & \frac{1}{2} a_{22} & a_{23} & a_{24} \\
a_{13} & a_{23} & \frac{1}{2} a_{33} & a_{34} \\
a_{14} & a_{24} & a_{34} & \frac{1}{2} a_{44}
\end{array}\right]
$$

If matrix $\mathrm{A}$ is positive definite, then $V(x)$ is positive definite. Sylvester's criterion [19] used to determine matrix $\mathrm{A}$ is positive definite.

$$
\begin{aligned}
\Delta_{1} & =\frac{1}{2} a_{11}>0, a_{11}>0 \\
\Delta_{2} & =\left|\begin{array}{cc}
\frac{1}{2} a_{11} & a_{12} \\
a_{12} & \frac{1}{2} a_{22}
\end{array}\right|=\left(\frac{1}{2} a_{11} \cdot \frac{1}{2} a_{22}\right)-\left(a_{12}-a_{12}\right) \\
& =\frac{1}{4} a_{11} \cdot a_{22}-a_{12}^{2}>0 \\
\Delta_{3} & =\left|\begin{array}{lll}
\frac{1}{2} a_{11} & a_{12} & a_{13} \\
a_{12} & \frac{1}{2} a_{22} & a_{23} \\
a_{13} & a_{23} & \frac{1}{2} a_{33}
\end{array}\right|=\frac{1}{2} a_{11}\left|\begin{array}{cc}
\frac{1}{2} a_{22} & a_{23} \\
a_{23} & \frac{1}{2} a_{33}
\end{array}\right|
\end{aligned}
$$


"Dynamical System Stability Analysis of the Interaction Nitrogen, Phytoplankton, Macrobenthos, and Sediment with Gradient Variable Method"

$$
\begin{aligned}
& -a_{12}\left|\begin{array}{cc}
a_{12} & a_{23} \\
a_{13} & \frac{1}{2} a_{33}
\end{array}\right|+a_{13}\left|\begin{array}{cc}
a_{12} & \frac{1}{2} a_{22} \\
a_{13} & a_{23}
\end{array}\right| \\
& =\frac{1}{8} a_{11} \cdot a_{22} \cdot a_{33}-\frac{1}{2} a_{11} \cdot a_{23}^{2}-\frac{1}{2} a_{33} \cdot a_{12}^{2} \\
& +2 a_{12} \cdot a_{23} \cdot a_{13}-\frac{1}{2} a_{22} \cdot a_{13}^{2}>0 \\
& \Delta_{4}=\left|\begin{array}{cccc}
\frac{1}{2} a_{11} & a_{12} & a_{13} & a_{14} \\
a_{12} & \frac{1}{2} a_{22} & a_{23} & a_{24} \\
a_{13} & a_{23} & \frac{1}{2} a_{33} & a_{34} \\
a_{14} & a_{24} & a_{34} & \frac{1}{2} a_{44}
\end{array}\right| \\
& =\frac{1}{2} a_{11}\left|\begin{array}{ccc}
\frac{1}{2} a_{22} & a_{23} & a_{24} \\
a_{23} & \frac{1}{2} a_{33} & a_{34} \\
a_{24} & a_{34} & \frac{1}{2} a_{44}
\end{array}\right|-a_{12}\left|\begin{array}{ccc}
a_{12} & a_{23} & a_{24} \\
a_{13} & \frac{1}{2} a_{33} & a_{34} \\
a_{14} & a_{34} & \frac{1}{2} a_{44}
\end{array}\right| \\
& +a_{13}\left|\begin{array}{ccc}
a_{12} & \frac{1}{2} a_{22} & a_{24} \\
a_{13} & a_{23} & a_{34} \\
a_{14} & a_{24} & \frac{1}{2} a_{44}
\end{array}\right|-a_{14}\left|\begin{array}{ccc}
a_{12} & \frac{1}{2} a_{22} & a_{23} \\
a_{13} & a_{23} & \frac{1}{2} a_{33} \\
a_{14} & a_{24} & a_{34}
\end{array}\right| \\
& =\frac{1}{16} a_{11} a_{22} a_{33} a_{44}-\frac{1}{4} a_{11} a_{22} a_{34}^{2}-\frac{1}{4} a_{11} a_{23}^{2} a_{44} \\
& +a_{11} a_{23} a_{24} a_{34}-\frac{1}{4} a_{11} a_{33} a_{24}^{2}-\frac{1}{4} a_{12}^{2} a_{33} a_{44} \\
& +a_{12} a_{22} a_{34} a_{14}+a_{12}^{2} a_{34}^{2}-2 a_{12} a_{23} a_{34} a_{14} \\
& +a_{12} a_{23} a_{13} a_{44}-2 a_{12} a_{24} a_{13} a_{34}+a_{12} a_{24} a_{33} a_{14} \\
& -\frac{1}{4} a_{22} a_{13}^{2} a_{44}+a_{13}^{2} a_{24}^{2}-2 a_{13} a_{24} a_{23} a_{14} \\
& -\frac{1}{4} a_{22} a_{33} a_{14}^{2}+a_{23}^{2} a_{14}^{2}>0 \\
& \text { If } a_{11}, a_{12}, a_{13}, a_{14}>0, a_{21}, a_{22}, a_{23}, a_{24}>0 \\
& a_{31}, a_{32}, a_{33}, a_{34}>0, a_{41}, a_{42}, a_{43}, a_{44}>0 \text {, then } V(x) \text { is }
\end{aligned}
$$

\begin{tabular}{|c|c|c|c|c|}
\hline Symbol & $\begin{array}{l}\text { Definition of } \\
\text { Parameter }\end{array}$ & Value & Dimension & Source \\
\hline \multirow[t]{2}{*}{$\mu$} & Sedimentation & & & \\
\hline & $\begin{array}{l}\text { rate of } \\
\text { phytoplankton }\end{array}$ & 0.0005 & day $^{-1}$ & [estimated] \\
\hline$\gamma$ & $\begin{array}{l}\text { Water } \\
\text { exchange rate }\end{array}$ & 7.0113 & day $^{-1}$ & [estimated] \\
\hline$\tau$ & $\begin{array}{l}\text { Sedimentation } \\
\text { rate of } \\
\text { macrobenthos }\end{array}$ & 0.0024 & day $^{-1}$ & [estimated] \\
\hline$\omega$ & $\begin{array}{l}\text { Proportion of } \\
\text { the } \\
\text { remineralizatio } \\
n \text { rate of } \\
\text { nitrogen }\end{array}$ & $\begin{array}{l}16.505 \\
7\end{array}$ & - & [estimated] \\
\hline$\alpha$ & $\begin{array}{l}\text { Maximum } \\
\text { growth rate of } \\
\text { phytoplankton }\end{array}$ & $\begin{array}{l}6.5958 \\
.10^{-9}\end{array}$ & day $^{-1}$ & [estimated] \\
\hline$\beta$ & $\begin{array}{l}\text { Total mortality } \\
\text { of } \\
\text { phytoplankton }\end{array}$ & 2.0419 & - & [estimated] \\
\hline$K_{N}$ & $\begin{array}{l}\text { Constant half- } \\
\text { saturation of } \\
\text { nitrogen }\end{array}$ & 0.0207 & $\mathrm{~g} / \mathrm{m}^{3}$ & [21] \\
\hline A & $\begin{array}{l}\text { Input the } \\
\text { remaining feed } \\
\text { and excretion } \\
\text { of shrimp for } \\
\text { nitrogen }\end{array}$ & 6.1330 & $\mathrm{mg} \mathrm{g}_{1}^{-1}$ day $^{-}$ & [estimated] \\
\hline
\end{tabular}

positive definite. Thus, the system of eqution (5) is globally asymptotically stable.

\section{NUMERICAL SIMULATIONS}

Based on data that obtained in the shrimp pond BBPBAP Jepara, value of the parameters in the system model of equation (5) is given. Numerical simulations are used for the analysis of dynamical system. Parameter values obtained by using the non-linear least square method [20]. The following parameters are given in TABLE 1.
Substitute the parameter values into equation (5) so that the following equation is obtained

$$
\begin{aligned}
& \dot{P}=\frac{6.5958 \times 10^{-9} N P}{0.0207+N}-9.0532 P \\
& \dot{N}=6.1338+16.5057 S-7.0113 N-\frac{6.5958 \times 10^{-9} N P}{0.0207+N} \\
& \dot{M}=2.0409 P-7.0137 M \\
& \dot{S}=9.8492 \times 10^{-4} P+0.0024 M-16.5057 S
\end{aligned}
$$

where $\mathrm{P}$ is phytoplankton, $\mathrm{N}$ is nitrogen, $\mathrm{M}$ is macrobenthos, and $\mathrm{S}$ is sediment at $t$ is time with equilibrium point:

$e^{*}=(P, N, M, S)=(0,0.8748,0,0)$

Linear equation depending on the time :

$$
\begin{aligned}
\dot{x} & =f(x, t)=\left[\begin{array}{c}
\dot{P} \\
\dot{N} \\
\dot{M} \\
\dot{S}
\end{array}\right], \text { so that obtained } \\
f & =\left[\begin{array}{l}
f_{1} \\
f_{2} \\
f_{3} \\
f_{4}
\end{array}\right]=\left[\begin{array}{c}
\frac{6.5958 \times 10^{-9} N P}{0.0207+N}-9.0532 P \\
6.1330+16.5057 S-7.0113 N-\frac{6.5958 \times 10^{-9} N P}{0.0207+N} \\
2.0409 P-7.0137 M \\
9.8492 \times 10^{-4} P+0.0024 M-16.5057 S
\end{array}\right]
\end{aligned}
$$

Assume an arbitrary $\nabla V$ 
"Dynamical System Stability Analysis of the Interaction Nitrogen, Phytoplankton, Macrobenthos, and Sediment with Gradient Variable Method"

$\nabla V(x)=\left[\begin{array}{l}a_{11} P+a_{12} N+a_{13} M+a_{14} S \\ a_{21} P+a_{22} N+a_{23} M+a_{24} S \\ a_{31} P+a_{32} N+a_{33} M+a_{34} S \\ a_{41} P+a_{42} N+a_{43} M+a_{44} S\end{array}\right]$,

Then from equation (8), $\bar{V}$ can be determined from $\nabla V$ (gradient of $V$ ) as follows

$\bar{V}(x)=\nabla V^{T} f$

$$
\begin{gathered}
\dot{V}(x)=\left[\begin{array}{c}
a_{11} P+a_{12} N+a_{13} M+a_{14} S \\
a_{21} P+a_{22} N+a_{23} M+a_{24} S \\
a_{31} P+a_{32} N+a_{33} M+a_{34} S \\
a_{41} P+a_{42} N+a_{43} M+a_{44} S
\end{array}\right]^{T} \\
{\left[\begin{array}{c}
\frac{6.5958 \times 10^{-9} N P}{0.0207+N}-9.0532 P \\
6.1330+16.5057 S-7.0113 N-\frac{6.5958 \times 10^{-9} N P}{0.0207+N} \\
2.0409 P-7.0137 M \\
9.8492 \times 10^{-4} P+0.0024 M-16.5057 S
\end{array}\right]}
\end{gathered}
$$$$
=\left(a_{11} P+a_{12} N+a_{13} M+a_{14} S\right)\left(\frac{6.5958 \times 10^{-9} N P}{0.0207+N}-9.0532 P\right)
$$$$
+\left(a_{21} P+a_{22} N+a_{23} M+a_{24} S\right)
$$$$
\left(6.1330+16.5057 S-7.0113 N-\frac{6.5958 \times 10^{-9} N P}{0.0207+N}\right)
$$$$
+\left(a_{31} P+a_{32} N+a_{33} M+a_{34} S\right)(2.0409 P-7.0137 M)
$$$$
+\left(a_{41} P+a_{42} N+a_{43} M+a_{44} S\right)
$$$$
\left(9.8492 \times 10^{-4} P+0.0024 M-16.5057 S\right)
$$

That obtained $V(x)$ is negative definite function if $a_{11}, a_{12}, a_{13}, a_{14}>0, a_{21}, a_{22}, a_{23}, a_{24}>0$

$a_{31}, a_{32}, a_{33}, a_{34}>0, a_{41}, a_{42}, a_{43}, a_{44}>0$

The qurl equations are

$$
\begin{array}{ll}
\frac{\partial \nabla V_{1}}{\partial N}=\frac{\partial \nabla V_{2}}{\partial P} \Rightarrow a_{12}=a_{21} & \frac{\partial \nabla V_{1}}{\partial M}=\frac{\partial \nabla V_{4}}{\partial P} \Rightarrow a_{14}=a_{41} \\
\frac{\partial \nabla V_{1}}{\partial F}=\frac{\partial \nabla V_{3}}{\partial P} \Rightarrow a_{13}=a_{31} & \frac{\partial \nabla V_{3}}{\partial M}=\frac{\partial \nabla V_{4}}{\partial F} \Rightarrow a_{34}=a_{43} \\
\frac{\partial \nabla V_{2}}{\partial F}=\frac{\partial \nabla V_{3}}{\partial N} \Rightarrow a_{23}=a_{32} & \frac{\partial \nabla V_{2}}{\partial M}=\frac{\partial \nabla V_{4}}{\partial N} \Rightarrow a_{24}=a_{42}
\end{array}
$$

The qurl equations are satisfied, so the $V$ can be computed as

$V(x)=\int \nabla V \cdot d x$

$=\int_{0}^{P} \nabla V_{1} \cdot d P+\int_{0}^{N} \nabla V_{2} \cdot d N+\int_{0}^{M} \nabla V_{3} \cdot d M+\int_{0}^{S} \nabla V_{4} \cdot d S$

$$
=\int_{0}^{P} a_{11} P+a_{12} N+a_{13} M+a_{14} S . d P
$$

$+\int_{0}^{N} a_{21} P+a_{22} N+a_{23} M+a_{24} S . d N$

$$
\begin{aligned}
& +\int_{0}^{M} a_{31} P+a_{32} N+a_{33} M+a_{34} S . d M \\
& +\int_{0}^{S} a_{41} P+a_{42} N+a_{43} M+a_{44} S . d S \\
& =\frac{1}{2} a_{11} P^{2}+a_{12} N P+a_{13} M P+a_{14} S P \\
& +\frac{1}{2} a_{22} N^{2}+a_{21} P N+a_{23} M N+a_{24} S N \\
& \quad+\frac{1}{2} a_{33} M^{2}+a_{31} P M+a_{32} N M+a_{34} S M \\
& \quad+\frac{1}{2} a_{44} S^{2}+a_{41} P S+a_{42} N S+a_{43} M S \\
& \quad V(x)=\frac{1}{2} a_{11} P^{2}+\frac{1}{2} a_{22} N^{2}+\frac{1}{2} a_{33} M^{2}+\frac{1}{2} a_{44} S^{2} \\
& +2 a_{12} P N+2 a_{13} P M+2 a_{14} P S \\
& +2 a_{23} M N+2 a_{24} S N+2 a_{34} S M
\end{aligned}
$$

Then, determined the positive definite matrix equation for the Lyapunov function as follows

$$
\begin{aligned}
V(x)= & x^{T} P x \\
= & {\left[\begin{array}{llll}
P & N & M & S
\end{array}\right]\left[\begin{array}{c}
A_{11} P+A_{12} N+A_{13} M+A_{14} S \\
A_{21} P+A_{22} N+A_{23} M+A_{24} S \\
A_{31} P+A_{32} N+A_{33} M+A_{34} S \\
A_{41} P+A_{42} N+A_{43} M+A_{44} S
\end{array}\right] } \\
V(x)= & A_{11} P^{2}+A_{12} P N+A_{13} P M+A_{14} P S \\
& +A_{22} N^{2}+A_{21} N P+A_{23} N M+A_{24} N S \\
& +A_{33} M^{2}+A_{31} M P+A_{32} M N+A_{34} M S \\
& +A_{44} S^{2}+A_{41} S P+A_{42} S N+A_{43} S M \\
V(x)= & A_{11} P^{2}+A_{22} N^{2}+A_{33} M+A_{44} S^{2}+2 A_{12} P N \\
+ & 2 A_{13} P M+2 A_{14} P S+2 A_{23} N M \\
+ & 2 A_{24} N S+2 A_{34} M S
\end{aligned}
$$

Equations (10) and (11) are same, so we have

$$
\begin{aligned}
& V(x)= \frac{1}{2} a_{11} P^{2}+\frac{1}{2} a_{22} N^{2}+\frac{1}{2} a_{33} M^{2}+\frac{1}{2} a_{44} S^{2} \\
&+2 a_{12} P N+2 a_{13} P M+2 a_{14} P S \\
&+2 a_{23} M N+2 a_{24} S N+2 a_{34} S M \\
&=A_{11} P^{2}+A_{22} N^{2}+A_{33} M^{2}+A_{44} S^{2} \\
&+2 A_{12} P N+2 A_{13} P M+2 A_{14} P S \\
&+2 A_{23} N M+2 A_{24} N S+2 A_{34} M S
\end{aligned}
$$

Than we obtained

$A_{11}=\frac{1}{2} a_{11} ; A_{22}=\frac{1}{2} a_{22} ; A_{33}=\frac{1}{2} a_{33} ; A_{44}=\frac{1}{2} a_{44} ;$

$A_{12}=a_{12} ; A_{13}=a_{13} ; A_{14}=a_{14} ; A_{23}=a_{23} ; A_{24}=a_{24} ; A_{34}=a_{34}$

So, we obtained matrix $\mathrm{A}$ 
"Dynamical System Stability Analysis of the Interaction Nitrogen, Phytoplankton, Macrobenthos, and Sediment with Gradient Variable Method"

$A=\left[\begin{array}{cccc}\frac{1}{2} a_{11} & a_{12} & a_{13} & a_{14} \\ a_{12} & \frac{1}{2} a_{22} & a_{23} & a_{24} \\ a_{13} & a_{23} & \frac{1}{2} a_{33} & a_{34} \\ a_{14} & a_{24} & a_{34} & \frac{1}{2} a_{44}\end{array}\right]$

If matrix A is positive definite, then $V(x)$ positive definite. Sylvester's criterion [19] is used to determine matrix A positive definite.

$$
\begin{aligned}
& \Delta_{1}= \frac{1}{2} a_{11}>0, a_{11}>0 \\
& \Delta_{2}=\left|\begin{array}{ll}
\frac{1}{2} a_{11} & a_{12} \\
a_{12} & \frac{1}{2} a_{22}
\end{array}\right|=\left(\frac{1}{2} a_{11} \cdot \frac{1}{2} a_{22}\right)-\left(a_{12}-a_{12}\right) \\
&= \frac{1}{16} a_{11} a_{22} a_{33} a_{44}-\frac{1}{4} a_{11} a_{22} a_{34}^{2}-\frac{1}{4} a_{11} a_{23}^{2} a_{44} \\
&+a_{11} a_{23} a_{24} a_{34}-\frac{1}{4} a_{11} a_{33} a_{24}^{2}-\frac{1}{4} a_{12}^{2} a_{33} a_{44} \\
&+a_{12} a_{22} a_{34} a_{14}+a_{12}^{2} a_{34}^{2}-2 a_{12} a_{23} a_{34} a_{14} \\
&+a_{12} a_{23} a_{13} a_{44}-2 a_{12} a_{24} a_{13} a_{34}+a_{12} a_{24} a_{33} a_{14} \\
&-\frac{1}{4} a_{22} a_{13}^{2} a_{44}+a_{13}^{2} a_{24}^{2}-2 a_{13} a_{24} a_{23} a_{14} \\
&-\frac{1}{4} a_{22} a_{33} a_{14}^{2}+a_{23}^{2} a_{14}^{2}>0 \\
& \text { If } a_{11}, a_{12}, a_{13}, a_{14}>0, a_{21}, a_{22}, a_{23}, a_{24}>0
\end{aligned}
$$

$a_{31}, a_{32}, a_{33}, a_{34}>0, a_{41}, a_{42}, a_{43}, a_{44}>0$. Then $V(x)$ is positive definite. Based Sylvester's criterion obtained matrix A, as follows:

$$
A=\left[\begin{array}{llll}
4 & 2 & 2 & 2 \\
2 & \frac{3}{2} & 1 & 1 \\
2 & 1 & 5 & 1 \\
2 & 1 & 1 & \frac{3}{2}
\end{array}\right]
$$

Based on equations (12), we obtained $V(x)$

$$
\begin{aligned}
V(x)= & \frac{1}{2} a_{11} P^{2}+\frac{1}{2} a_{22} N^{2}+\frac{1}{2} a_{33} M^{2}+\frac{1}{2} a_{44} S+2 a_{12} P N+2 a_{13} P M \\
& +2 a_{14} P S+2 a_{23} M N+2 a_{24} S N+2 a_{34} S M^{2} \\
= & 4 P^{2}+\frac{3}{2} N^{2}+5 M^{2}+\frac{3}{2} S+4 P N \\
& +4 P M+4 P S+2 M N+2 S N+2 S M^{2}
\end{aligned}
$$

Substitute the equilibrium point $e^{*}=(P, N, M, S)$ $=(0,0.8748,0,0), \quad$ so $\quad$ we obtained $\quad V(x)=1.1479 \geq 0$ (positive definite).

Meanwhile, based on equation (9)

$$
\begin{gathered}
\dot{V}(x)=\left(a_{11} P+a_{12} N+a_{13} M+a_{14} S\right)\left(\frac{6.5958 \times 10^{-9} N P}{0.0207+N}-9.0532 P\right) \\
+\left(a_{21} P+a_{22} N+a_{23} M+a_{24} S\right)
\end{gathered}
$$

$$
\begin{aligned}
& \left(6.1338+16.5057 S-7.0113 N-\frac{6.5958 \times 10^{-9} N P}{0.0207+N}\right) \\
& +\left(a_{31} P+a_{32} N+a_{33} M+a_{34} S\right)(2.0409 P-7.0137 M) \\
& +\left(a_{41} P+a_{42} N+a_{43} M+a_{44} S\right) \\
& \left(9.8492 \times 10^{-4} P+0.0024 M-16.5057 S\right) \\
\dot{V}(x)= & (8 P+2 N+2 M+2 S)\left(\frac{6.5958 \times 10^{-9} N P}{0.0207+N}-9.0532 P\right) \\
+ & (2 P+3 N+M+S)\left(6.1330+16.5057 S-7.0113 N-\frac{6.5958 \times 10^{-9} N P}{0.0207+N}\right) \\
+ & (2 P+N+10 M+S)(2.0409 P-7.0137 M) \\
+ & (2 P+N+M+3 S)\left(9.8492 \times 10^{-4} P+0.0024 M-16.5057 S\right)
\end{aligned}
$$

Substitute the equilibrium point for

$e^{*}=(P, N, M, S)=(0,0.8748,0,0)$

so we obtained $\dot{V}(x)=-1.3122 \times 10^{-3} \leq 0$ (negative definite).

It is proven that $V(x)=1.1479 \geq 0$ is positive definite and $\dot{V}(x)=-1.3122 \times 10^{-3} \leq 0$ is negative definite. Thus, the system of eqution (8) is globally asymptotically stable. This shows that the dynamical system of the shrimp ponds is stable and has a good water quality for cultivation.

The following are figures of the dynamical phytoplankton (P), nitrogen concentration (N), macrobenthos (M), and sediment (S) with MATLAB software.

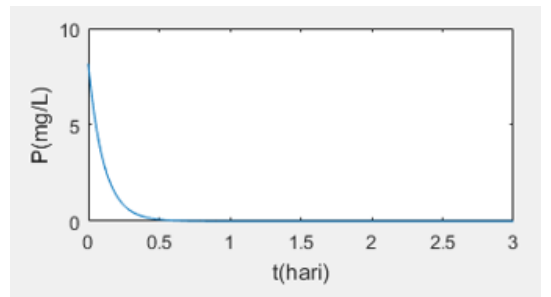

Figure 2. Dynamical Phytoplankton (P)

Figure 2 shows the change in the abundance of phytoplankton $(\mathrm{P})$ for 3 days with the parameter values in table 1 and the initial value of $\mathrm{P}(0)=8,167 \mathrm{mg} / \mathrm{L}$. The graph of the phytoplankton population moves down and reaches the equilibrium point of 0 .

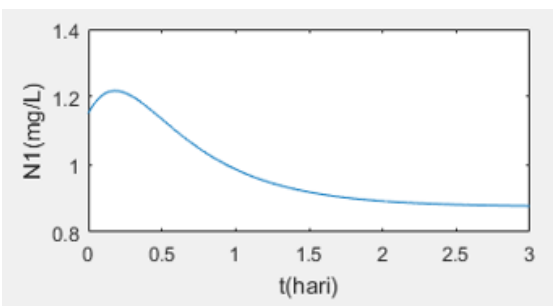

Figure 3. Dynamical Nitrogen Concentration (N)

Figure 3 shows the change in nitrogen concentration for 3 days with an initial value of $\mathrm{N}(0)=1.15135 \mathrm{mg} / \mathrm{L}$. At this initial value, the dynamical graph of nitrogen reaches a maximum peak of $1.2 \mathrm{mg} / \mathrm{L}$. Then after 1 day, the graph 
gradually decreased and reached a concentration of 0.8748 $\mathrm{mg} / \mathrm{L}$.

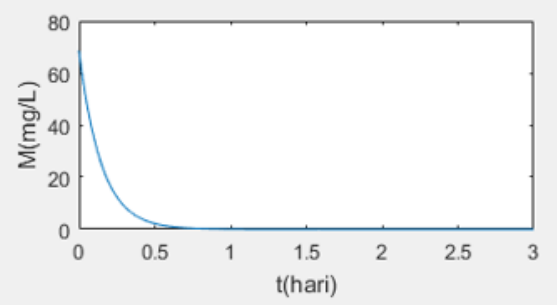

Figure 4. Dynamical Macrobenthos (M)

Figure 4 shows the change in the population of macrobenthos (M) for 3 days with the parameter values in table 1 and the initial value of $\mathrm{M}(0)=68,571 \mathrm{mg} / \mathrm{L}$. The graph of the macrobenthos population moves down and reaches the equilibrium point of 0 .

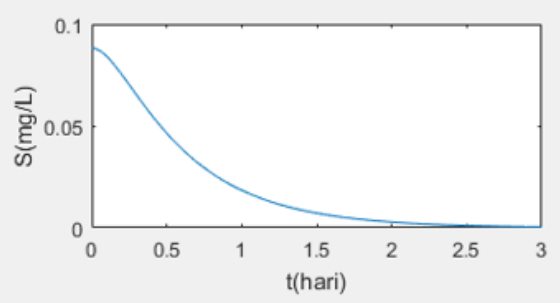

Figure 5. Dynamical Sediment (S)

Figure 5 shows the change in the total sediment population (S) for 3 days with the parameter values in table 1 and the initial value $\mathrm{S}(0)=0.08812 \mathrm{mg} / \mathrm{L}$. The graph of the macrobenthos population moves down and reaches the equilibrium point of 0 after 1 day water conservancy.

Based on figures 2,4,5 the dynamical graph of phytoplankton, macrobenthos, and sediment are closed to 0 equilibrium point. This shows that after 1 day of pond water maintenance, the abundance of phytoplankton, macrobenthos density, and sediment is almost non-existent because water in the pond is changed every day. While on figure 3 the nitrogen concentration stable at 0.8748 . This means that the water quality is still within the standard limits for shrimp pond.

\section{CONCLUSION}

In this paper has been developed a mathematical model of dynamical system in aquaculture of shrimp pond by adding a macrobenthos variable. This dynamical system model is a nonlinear system of differential equations with four variables. The global stability analysis of dynamical system obtained by constructing the Lyapunov function with gradient variable method. Based on numerical simulation, we obtained that globally asymptotically stable at equilibrium point $(P, N, M, S)=(0,0.8748,0,0)$. It showed that the water quality is globally asymptotically stable with phytoplankton, macrobenthos, and sediment approaching the equilibrium point zero, while the equilibrium point concentrate of nitrogen is $0.8748 \mathrm{mg} / \mathrm{L}$. This indicated that the quality of the shrimp pond in BBPBAP Jepara Indonesia was still considerably in normal condition for shrimp farming and there are no changes in biotic and abiotic variables that can disrupt the stability of aquatic ecosystems.

\section{ACKNOWLEDGMENTS}

The authors would like to thank the Directorate General of Higher Education (DIKTI) for financially supporting this research as Matching Fund-Kedaireka program with contract number 2181/E3/PKS.06/KL/2021. This research is also supported by Mathematical Modeling and Computer Laboratory, Department of Mathematics and Center of Marine Ecology and Biomonitoring for Sustainable Aquaculture (Ce-MEBSA), Diponegoro University, Semarang.

\section{REFERENCES}

1. I. Arismendi and B. E. Penaluna. 2016. "Examining Diversity Inequities in Fisheries Science : A Call to Action," vol. 66, no. 7, pp. 584 591, doi: 10.1093/biosci/biw041.

2. C. To, F. Security, and N. F. O. R. All. 2016. THE STATE OF WORLD FISHERIES AND AQUACULTURE.

3. J. Cardoso-mohedano et al., 2016. "Science of the Total Environment Reducing nutrient impacts from shrimp ef fl uents in a subtropical coastal lagoon," Sci. Total Environ., vol. 571, no. July, pp. 388-397, 2016, doi: 10.1016/j.scitotenv.06.140.

4. P. Yang, D. Y. F. Lai, B. Jin, D. Bastviken, L. Tan, and C. Tong. 2017. "Science of the Total Environment Dynamics of dissolved nutrients in the aquaculture shrimp ponds of the Min River estuary, China: Concentrations, fl uxes and environmental loads," Sci. Total Environ., vol. 603-604, pp. 256-267, 2017, doi: 10.1016/j.scitotenv. 06.074.

5. J. A. Hargreaves. 1997. "A simulation model of ammonia dynamics in commercial catfish ponds in the southeastern United States," vol. 16, pp. 27-43.

6. K. Lorenzen and J. Struve, 1997. "Impact of farming intensity and water management on nitrogen dynamics in intensive pond culture: a mathematical model applied to Thai commercial shrimp farms," pp. 493-507.

7. A. Meuret. 2001. "Modeling approach of nitrogen and phosphorus exchanges at the sediment - water interface of an intensive fishpond system,"

8. L. E. Meijer and Y. Avnimelech. 1999. "On the use of micro-electrodes in fish pond sediments," vol. 21, pp. 71-83.

9. M. A. Burford and A. R. Longmore. 2001. "High ammonium production from sediments in hypereutrophic shrimp ponds," vol. 224, pp. 187- 
195.

10. M. A. Burford and K. Lorenzen. 2004. "Modeling nitrogen dynamics in intensive shrimp ponds: The role of sediment remineralization," Aquaculture, vol. 229 , no. 1-4, pp. 129-145, doi: 10.1016/S0044-8486(03)00358-2.

11. U. F. Architecture, M. A. Romli, S. Daud, Y. Qian, and H. Zhang. 2018. "Modeling of nitrogen transformation in an integrated multi-trophic aquaculture (IMTA) Modeling of nitrogen transformation in an integrated multi- trophic aquaculture (IMTA),"

12. E. Triyana, Widowati, S. P. Putro. 2020. "Globally stability analysis of the mathematical model in the IMTA system by using the energy-Casimir method Globally stability analysis of the mathematical model in the IMTA system by using the energyCasimir method," doi: 10.1088/17426596/1524/1/012052.

13. L. F. Ribeiro, G. F. Eça, F. Barros, and V. Hatje, 2015. "Impacts of shrimp farming cultivation cycles on macrobenthic assemblages and chemistry of sediments *," Environ. Pollut., vol. 211, pp. 307-315, doi: 10.1016/j.envpol. 12.031.

14. S. P. Putro. 2014. Metode Sampling Penelitian Makrobenthos dan Aplikasinya. Semarang: Graha Ilmu.

15. Ittok Rochmad Choirudin, Mustofa Niti Supardjo, and Max Rudolf Muskananfola. 2014. Studi Hubungan Kandungan Bahan Organik Sedimen dengan Kelimpahan Makrozoobenthos di Muara Sungai Wedung Kabupaten Demak, "http://ejournals1.undip.ac.id/index.php/maquares," vol. 3, pp. 168-176.

16. M. Ulfa, P. Gde, S. Julyantoro, A. Hermawati, and W. Sari. 2018. "Keterkaitan Komunitas Makrozoobentos dengan Kualitas Air dan Substrat di Ekosistem Mangrove Taman Hutan Raya Ngurah Rai Bali,” vol. 4, pp. 179-190.

17. R. Sundari, J. Matematika, F. Matematika, P. Alam, and A. S. Lorenz. 2017. "Konstruksi Fungsi Lyapunov untuk Menentukan Kestabilan," vol. 6, no. 1 , pp. 1-5.

18. Hassan K. Khalil. 2002. T. Edition, Nonlinear Systems Third Edition. Prentice-Hall, Inc, Upper Saddle River, NJ 07458.

19. X. Liao, L. Wang, and P. YU. 2007. "Stability of Dynamical System," Elsevier.

20. Didin Adri, 2016. A Note Lecturer "REGRESSION ANALYSIS IN NUMERICAL ANALYSIS WITH MAPLE”. Muhammadiyah Buton University-Baubau.

21. Y. Li, Y. Liu, L. Zhao, A. Hastings, and H. Guoaa. 2015. "Exploring Change of Internal Nutrients 